\section{Neuer Vorstand für AGNES}

Die Arbeitsgemeinschaft Neurodermitisschulung (AGNES) hat im Rahmen Ihrer Jahrestagung im vergangenen Februar in Würzburg einen neuen Vorstand gewählt Neue Präsidentin ist Priv.-Doz. Dr. Doris Staab von der Klinik für Pädiatrie

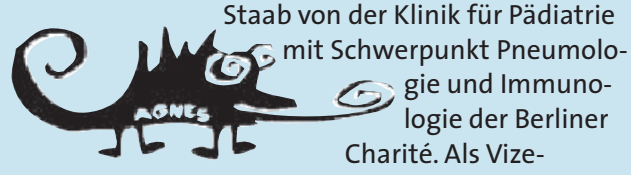
präsident bestätigt wurde Prof. Dr. Dr. Johannes Ring, Klinik für Dermatologie und Allergologie am Biederstein, Technische Universität München.

Die AGNES hat ein Schulungsprogramm entwickelt, das Patienten mit atopischem Ekzem hilft, mit ihrer Krankheit umzugehen. Dieses Schulungsprogramm wird in der Regel von den Krankenkassen bezahlt. Ein interdisziplinäres Team informiert und trainiert die Betroffenen und ggf. ihre Eltern. Weitere Informationen bietet die AGNES-Homepage unter www.neurodermitisschulung.de.

\section{Allergie im Stern}

Das Nachrichtenmagazin Stern hat auf seiner Homepage einen neuen eigenen Bereich zum Thema Allergie eingerichtet. Unter der Adresse http://stern.de/allergie finden sich umfassende laienverständliche Informationen, darunter ein interaktiver Symptomcheck, ein Expertenrat und eine Arztsuche. Für die fachliche Qualität steht das European Centre for Allergy Research Foundation (ECARF), Berlin, gerade.

\section{Patientenbroschüre Neurodermitis}

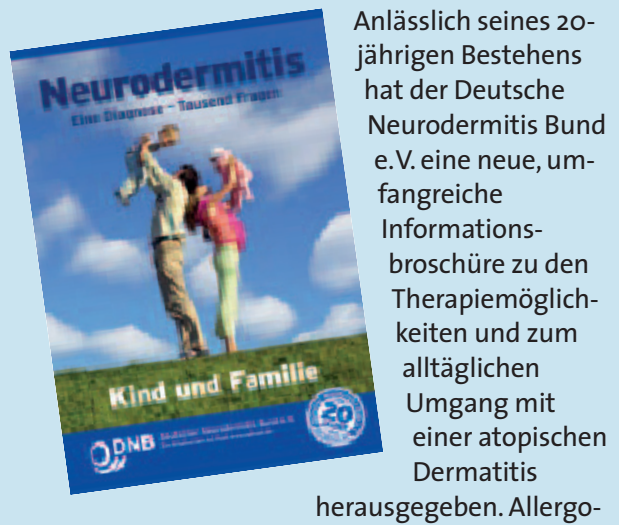

logen können das 92 Seiten starke Heft kostenlos zur Auslage im Wartezimmer bestellen per E-Mail an info@dnb-ev.de oder per Fax an (o 40) 231008.

\title{
Herxheimer-Preis geht nach Wien
}

m Rahmen des 19. Mainzer Allergie-

Workshops wurde am 16. März der Herbert-Herxheimer-Förderpreis 2007 der Deutschen Gesellschaft für Allergologie und klinische Immunologie (DGAKI) verliehen. Der Preis zeichnet jährlich herausragende Originalarbeiten aus, die sich mit diagnostischen und therapeutischen Fragestellungen oder der Grundlagenforschung auf den Gebieten der Allergologie und klinischen Immunologie befassen.

Dieses Jahr waren 15 Arbeiten aus 13 Instituten eingereicht worden, die Jury entschied sich für die Publikation Rauter I, Krauth MT, Flicker S, Gieras A, Westritschnig K, Vrtala S, Balic N, Spitzauer S, Huss-Marp J, Brockow K, Darsow U, Ring J, Behrendt H, Semper $\mathrm{H}$, Valent $\mathrm{P}$, Valenta R. Allergen cleavage by effector cell-derived proteases regulates allergic inflammation. FASEB J 2006; 20: 967-9. Erstautorin Mag. Dr. Ingrid Rauter vom Zentrum für Physiologie und Pathophysiologie am Institut für Pathophysiologie der medizinischen Universität Wien stellte in Mainz ihre Arbeit persönlich vor. Die Urkunde übergab DGAKI-Schatzmeister Dr. Claus Bachert. Mit der Auszeichnung verbunden ist eine Preissumme von $5.000 €$, zur Verfügung gestellt von der DPC Biermann, Bad Nauheim, seit 2006 Teil von Siemens Medical Solutions.

\section{Neue DGAKI-Satzung}

$\mathrm{D}$ ie Deutsche Gesellschaft für Allergologie und klinische Immunologie hat sich im Rahmen einer außerordentlichen Mitgliederversammlung am 16. März 2007 während des 19. Mainzer Allergie-Workshops eine neue Satzung gegeben. Eine Überarbeitung war nicht zuletzt deshalb erforderlich geworden, da die alte Satzung nicht mehr die aktuellen juristischen Vorgaben zur Anerkennung der Gemeinnützigkeit erfüllte.

Die neue Gesellschaftssatzung kann online unter der Adresse www.dgaki.de/ Satzung/Satzung-DGAKI-2007.pdf auf

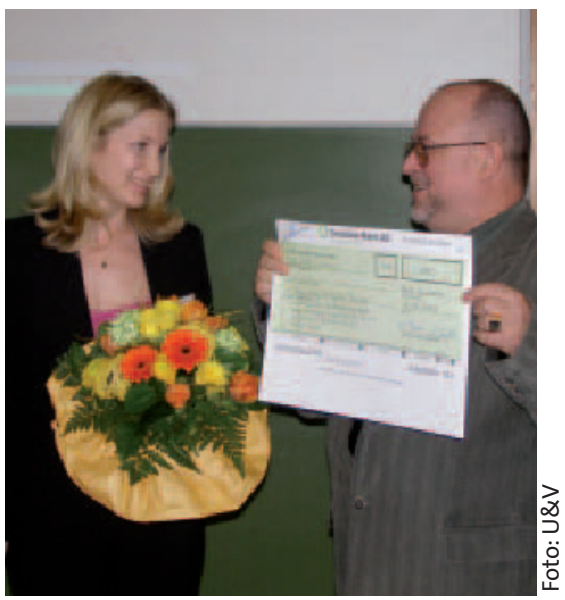

Herxheimer-Preisträgerin 2007 Mag. Dr. Ingrid Rauter erhält von Dr. Werner Kühnel, wissenschaftlicher Leiter bei DPC Biermann, den Scheck mit der Preissumme überreicht.

An der ausgezeichneten Publikation im Journal of the Federation of American Societies for Experimental Biology waren außer Wissenschaftlern der Universität Wien auch Forscher vom ZAUM - Zentrum für Allergie und Umwelt, von der klinischen Kooperationsgruppe Umweltdermatologie und Allergologie GSF/TUM der Technischen Universität München sowie von der Klinik und Poliklinik für Dermatologie und Allergologie am Biederstein beteiligt. 